\title{
A proposal of Extended ESG Considering ATSC Mobile DTV Environment
}

\author{
Ju Hyun Park and Jeong Hun Choi
}

\begin{abstract}
Current digital broadcasting service is providing various data service additionally along with general broadcasting service. A digital broadcasting service list and detailed information for various broadcasting service provide related information through Electronic Program Guide (EPG) and Electronic Service Guide (ESG). However, there are different problems to obtain ESG information through complicated information analysis process. This study provides a faster access method which is improved from current ESG for additional multi-media service in current broadcasting system. This proposed Extended-ESG can be applied to provide program-linked service and independent service through additional service access that is based on ATSC Mobile DTV, one-way or interactive broadcasting.
\end{abstract}

Index Terms-ESG (electronics service guide), extended ESG, SG (service guide) , ATSC mobile DTV, ATSC -M/H

\section{INTRODUCTION}

The fusion of broadcasting and telecommunication has brought about various kinds of high quality multimedia (Audio/Video, Image, Text, etc.) service through terrestrial, cable, satellite and mobile media. For mobile broadcasting service, different countries have different standards, for examples, Digital Multimedia Broadcasting (DMB), Digital Video Broadcasting-Handheld (DVB-H), and ATSC Mobile DTV (In other words, ATSC-Mobile/Handheld (M/H)).

A digital broadcasting system provides initial user interfaces such as EPG or ESG so that the user can choose the service of their want. EPG provides basic information about the current operated program whereas ESG provides detailed information for all the programs.

ATSC adopts Open Mobile Alliance-Mobile Broadcast Service Enabler Suite (OMA-BCAST)'s Service Guide (SG) user interface as standard because of the compatibility of mobile terminals $[1,2,3]$. ATSC M Mobile DTV transmits the necessary information for accessing the service in the form of Service Signalling Channel (SSC) of Fast Information Channel (FIC) and provides detailed information about the whole service in the form of SG [2, 3]. SG, which is based on OMA-BCAST, transmits metadata formed texts in IP data casting way using File Delivery over Unidirectional Transport (FLUTE) protocol. However, it is inefficient to receive SG through FLUTE protocol just to get additional service for the current program. It is because SG contains detailed information for all the programs and FLUTE requires complicated analysis stages of

Manuscript received March 15, 2012; revised May 5, 2012.

The authors are with Department of Electronics Engineering, Korea Polytechnic University, Jeongwang-dong, Siheung-City, Gyeonggi-do, Korea (e-mail: jhchoi@kpu.ac.kr). downloading. Therefore, this study provides faster and more efficient method for accessing additional multi-media service which meets the ATSC Mobile DTV standards.

This paper includes the following. In the 2nd chapter, ATSC Mobile DTV standards and SG will be investigated. In the 3rd chapter, Extended-ESG structure for accessing additional service for individual program will be provided and applicability of Extended-ESG in ATSC Mobile DTV environment will be analyzed. Lastly in the 4th chapter, a conclusion will be drawn regarding proposed Extended-ESG.

\section{RELATED RESEARCHES}

\section{A. ATSC Mobile DTV}

ATSC Mobile DTV is a mobile broadcasting technology which enables reception of video, sound, data service etc while moving from one place to another by transmitting mobile broadcasting stream in the spare bandwidth of $6 \mathrm{MHz}$ bandwidth of the standardization of Advanced Television Systems Committee, i.e. ATSC. In the standardization process of ATSC Mobile DTV, the compatibility with mobile phones, laptop computers, car TV and other mobile system was very important. As a result, as shown in Fig. 1, it is possible to transmit/receive all data with the application of IP protocol stack. In addition, to provide additional data service in IP protocol considering compatibility with mobile terminals, it also provides functional components such as OMA-BCAST SG, Service Content Protection (SCP) and OMA-Rich Media Environment (RME).

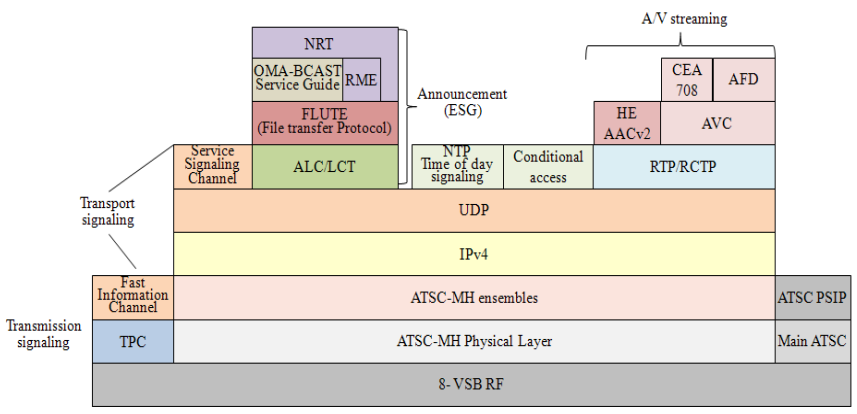

Fig. 1. ATSC Mobile DTV Protocol Stack Model

ATSC Mobile DTV system defines a new IP Stream called SSC which contains the minimum required information, to improve the access speed to service $[1,2]$. Through SSC, required information to access service such as broadcasting rating, encoding type and parameter and minimum information about the current program such as the title and time are transmitted. SSC is received through public IP Address (224.0.23.60) and Port number (4937) set by standards. The table section transmitted through SSC is 
shown in Table 1

TABLE 1: SSC TABLES OF ATSC MOBILE DTV

\begin{tabular}{|c|c|l|}
\hline $\begin{array}{c}\text { Table Name } \\
\begin{array}{c}\text { Service Map } \\
\text { Table (SMT- } \\
\text { MH) }\end{array}\end{array}$ & 0xDB & $\begin{array}{l}\text { List of services in Ensemble: Information } \\
\text { about each service. } \\
\text { List of components in each service with } \\
\text { Information about each component. } \\
\text { (analogous to VCT in standard A/65) }\end{array}$ \\
\hline $\begin{array}{c}\text { Guide Access } \\
\text { Table (GAT- } \\
\text { MH) }\end{array}$ & 0xDC & $\begin{array}{l}\text { Sources of Service Guide data: } \\
\text { Accommodates multiple SG data providers. } \\
\text { Gives provider name and Service ID of } \\
\text { each SG data service. }\end{array}$ \\
\hline $\begin{array}{c}\text { Cell Information } \\
\text { Table (CIT-MH) }\end{array}$ & 0xDD & $\begin{array}{l}\text { Supports hand-offs when roaming: } \\
\text { Supports hand-off between transmitters } \\
\text { when roming. } \\
\text { Tells about adjacent transmitters with } \\
\text { same/similar services }\end{array}$ \\
\hline $\begin{array}{c}\text { Service Labeling } \\
\text { Table (SLT-MH) }\end{array}$ & 0xDE & $\begin{array}{l}\text { Supports fast frequency scan: } \\
\text { Allows receivers to display useful service } \\
\text { list after brief RF scan. } \\
\text { Gives service names in one ensemble; no } \\
\text { need to visit all of them }\end{array}$ \\
\hline $\begin{array}{c}\text { Rating Region } \\
\text { Table (RRT) }\end{array}$ & 0xCA & $\begin{array}{l}\text { Supports Rating Region in broadcasting. } \\
\text { (RRT in Standard A/65) }\end{array}$ \\
\hline
\end{tabular}

One RS Frame of ATSC Mobile DTV contains at least one Service Map Table (SMT)-MH table section. SMT-MH contains all the service information of the selected ensemble. Each service is received distinguished by IP Multicast Address and UDP Port Number. Moreover, for decoding of each component, information of necessary encoding format and parameters is provided.

\section{B. General ESG}

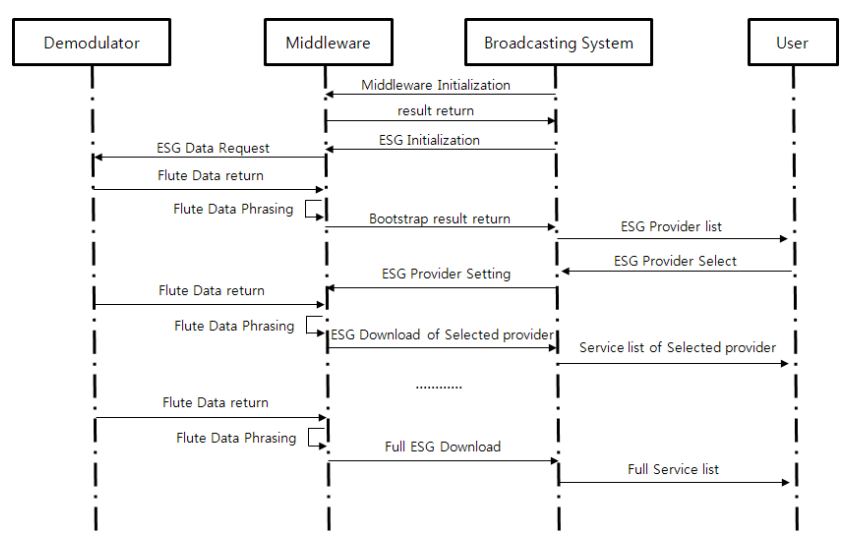

Fig. 2. ESG Download Procedure on Mobile Broadcast Terminal.

ESG data provides more detailed information on mobile TV than EPG which covers basic information such as program schedule and genres. Also, it enables viewers to choose service and contents according to their taste and it provides interface to access interactivity data. ESG is composed of metadata in the form of Extensible Mark-up Language (XML) text. It is transmitted as IP data stream which is compressed as Binary format for metadata (BIM) or GNU zip (GZIP) to minimize data amount. ESG data covers specific service provider and information about overall service such as service name, genre, program organization etc. Fig. 2 describes procedures to obtain ESG on a mobile reception device. It requires complicated analysis procedures because it provides analyzed information about the service provider or overall service.

\section{PROPOSED EXTENDED-ESG FOR THE ATSC MoBILE DTV}

\section{A. Proposed Extended-ESG}

ATSC Mobile DTV SG contains service access information about Service ID and Content ID(URL information in case of interactive broadcasting) to access additional service whether it is dependent on the program or not. In ATSC Mobile DTV standards, service access information about all the additional service is achieved through SG. However, in case when only access to additional service of current program is needed, it is inefficient in terms of data utility because it still requires time and cost to analyze all the unnecessary information. Therefore, it is noted that a method to access additional service of individual program without the analysis of SG is needed for faster service access.

In this study, Extended-ESG is proposed to enable faster access to basic additional service of individual program on SMT-MH of SSC. Service access information about individual program defines descriptor as Fig. 3 based on Component Descriptor Syntax and it is offered on Component Level of SMT-MH. The Extended-ESG Descriptor has the structure of the two types. It is defined considering real time one-way broadcasting and interactive broadcasting using return channel.

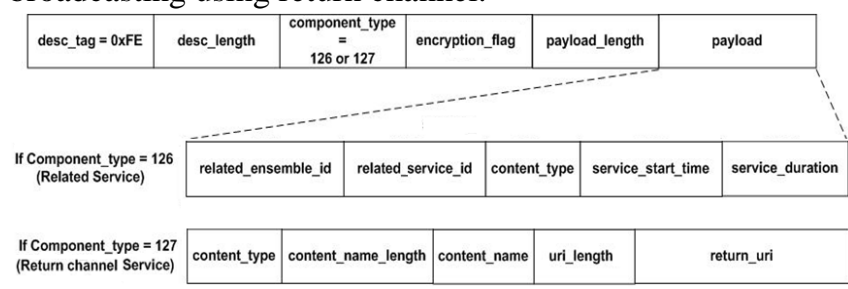

Fig. 3. Extended-ESG Descriptors

The proposed Extended-ESG uses Ensemble_id and Service_id field for one-way broadcasting network. It uses URL information for interactive broadcasting network and it offers location information of additional service for service linkage. Extended-ESG is providing additional service information about individual program and transmits them. The service form can be video, paused video clip, text and other forms that are defined by the service provider.

\section{B. Verification of Proposed Extended-ESG}

The test stream proposed in this study is created with the insertion of Extended-ESG information using current ATSC Mobile DTV broadcasting stream. The test stream is for ATSC Mobile DTV Multiplex Signal Generator (ATX2000) and validation of Extended-ESG basis.

ATSC Mobile DTV Browser is constructed with the insertion of Extended-ESG information analysis and additional multi-media service support function based on the Voice EPG SW [5] for ATSC Mobile DTV. It is realized in Windows PC environment considering the interlocking of wireless network for interactive broadcasting. 


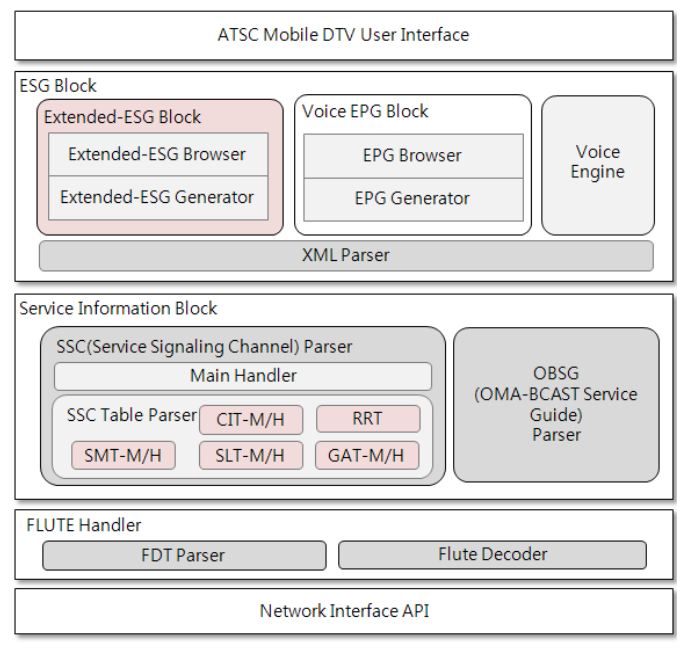

Fig. 4. Extended-ESG Browser Structure for ATSC Mobile DTV
ATSC Mobile DTV Browser analyses SSC and OBSG (OMA-BCAST Service Guide) in Service Information Block. In ESG Block, EPG and ESG information are provided based on the conducted analysis. Also information about Extended-ESG is obtained in SMT-MH. This enables the reception terminal to play various forms of service (Audio/Video, Image, Text, etc.) according to the content type about additional service transmitted by Extended-ESG Decoder.

On EPG Browser, if you choose one in the program list transmitted as ATSC Mobile DTV ensemble, it played related ATSC Mobile DTV program (video/audio). It also successfully played the contents after receiving Service_id, Ensemble_id or URL information (for linkage with return channel) transmitted by Extended-ESG Decoder. Also when asked for detailed information for all the programs, it downloaded FLUTE data through FLUTE and offered SG information based on the XML data analysis.

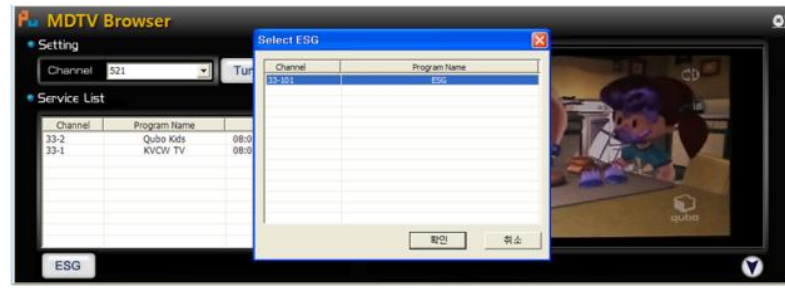

(a) ESG Selected

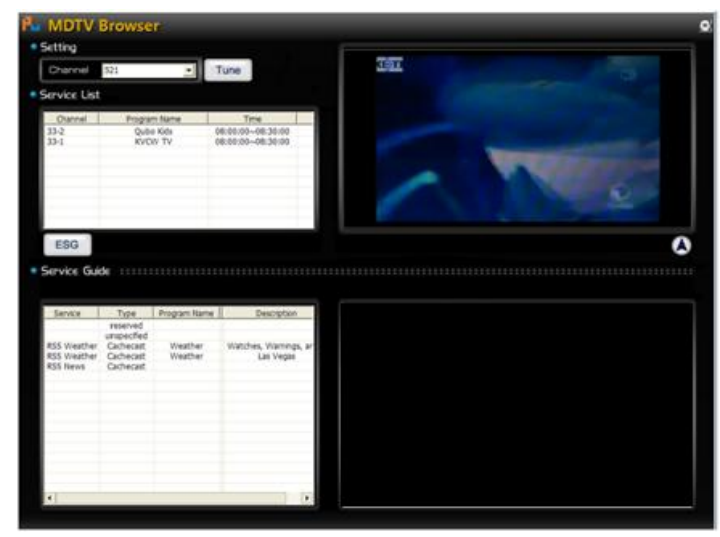

(b) ESG Selected result

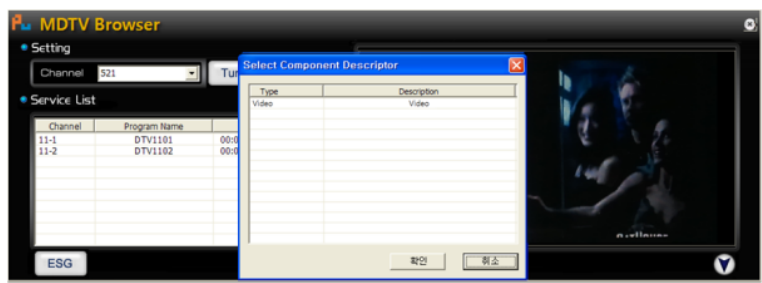

(c) Extended-ESG Selected

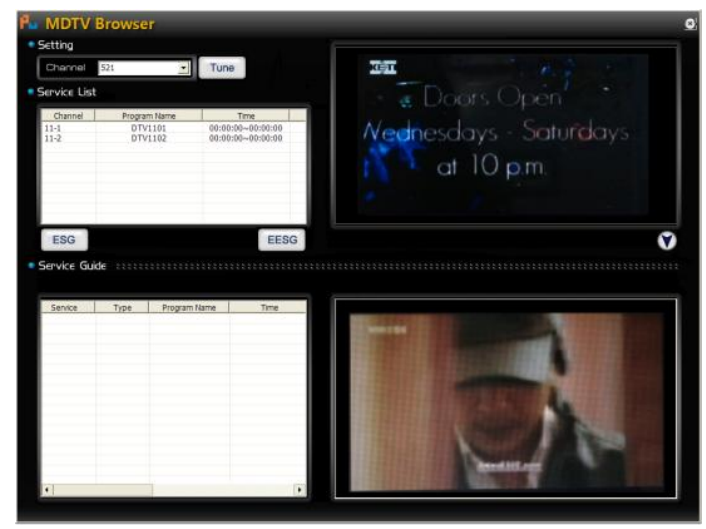

(d) Extended-ESG Selected result

Fig. 5. Extended-ESG Viewer

\section{CONCLUSION}

This study proposed Extended-ESG for offering faster additional service of the currently watched program in ATSC Mobile DTV environment. Also it was confirmed that it was possible to access additional service with SG analysis in ATSC Mobile DTV Browser through ExtendedESG Decoder and Extended-ESG Browser. The proposed Extended-ESG reduces time and cost consumed to access additional service of individual program. It only receives necessary information which can improve information utility. Also it takes real time one-way broadcasting and return channel for interactive broadcasting into account. This can be applied in providing new additional service for linked and non-linked broadcasting service.

In the future, research and development of structural method to provide service for real time broadcasting and multi-media service at the same time on a mobile screen.

\section{REFERENCES}

[1] ATSC: "ATSC Mobile DTV standard, Part1 - ATSC Mobile Digital Television System (A/153 Part1:2009)," ATSC standard, Document A/153 Part 1, 15 October 2009

[2] ATSC: "ATSC Mobile DTV Standard, Part 3 - Service Multiplex and Transport Subsystem Characteristics (A/153 Part3:2009)," ATSC standard, Document A/153 Part 3, 15 October 2009

[3] ATSC: "ATSC Mobile DTV Standard, Part 4 - Announcement(A/153 Part4:2009)," ATSC standard, Document A/153 Part 4, 15 October 2009

[4] ATSC: "ATSC Recommended Practice: Guide to the ATSC Mobile DTV Standard (Doc. A/154:2011), " ATSC standard, 15 April 2011

[5] C. Lim, J. Choi, J. Park, and N. Hwang, "Design And Implementation of Voice EPG SW Module for ATSC-MH," First Korea-Vietnam Technical Seminar on Information and Communications (TSIC 2010), KICS Vietnam Branch, Hanoi Vietnam, 2010. 12. 14 16

[6] J. Kim, S.-M. Jang, S.-I. Jeong, I.-S. Kyung, and H.-S. Kim, "Development of Service Guide for Mobile Broadcast Considering Interoperability," Korean Society of Broadcast Engineers Journal, Korean Society of Broadcast Engineers(KSBE), 2009. 11 